\title{
A modelagem matemática no monitoramento da criação de bezerras
}

\author{
Morgana Scheller \\ Instituto Federal Catarinense - Campus Rio do Sul \\ 89160-000, Rio do Sul, SC \\ E-mail: morganascheller@yahoo.com.br
}

\section{Rosana Maria Luvezute Kripka}

Universidade de Passo Fundo - Área de Matemática 99052-900, Campus I, BR 285, São José, Passo Fundo, RS

E-mail: rkripka@upf.br

\begin{abstract}
Resumo: O monitoramento da criação de bezerras é de fundamental importância para a otimização de lucros da produção leiteira, tendo em vista o alto custo para a obtenção e a manutenção de um rebanho sadio e produtivo. O processo de cria e recria é um constante desafio aos produtores, uma vez que o desenvolvimento rápido ou lento pode trazer prejuízos. Assim, torna-se importante o controle do crescimento das bezerras, considerando o peso e a altura ideais dos animais, de modo a obter-se a maior produtividade e rentabilidade possível. Segundo informações, disponíveis na literatura, para cada raça de futuras vacas leiteiras, existe um peso ideal para a primeira cobertura da bezerra, que ocorre por volta de 18 meses. Para o controle acompanhamento do peso dos animais, existem balanças calibradas próprias, no entanto, esses instrumentos, além de serem caros, também são de difícil manuseio, devido à necessidade de manejo e contenção do animal, o que também demanda tempo de trabalho específico. Assim, para contornar problemas dessa natureza, foram desenvolvidas fitas torácicas de pesagem, específicas para o acompanhamento do peso do animal. Tais fitas, contudo, nem sempre encontram-se disponíveis em agropecuárias. Tendo em vista essa problemática, propomos, neste artigo, uma modelagem matemática para obtenção de um modelo que estipule o peso aproximado em função do perímetro torácico da bezerra, que pode ser obtido por fita métrica comum. O objetivo deste trabalho é disponibilizar uma ferramenta computacional que possibilite obter o peso aproximado em função do comprimento do perímetro torácico, obtido com fita métrica comum, para facilitar o acompanhamento do controle do crescimento de bezerras.
\end{abstract}

Palavras- Chave: Modelagem matemática. Criação de bezerras. Modelo de crescimento exponencial. Perímetro torácico.

\section{Introdução}

$\mathrm{Na}$ atividade leiteira, para que ocorra a otimização dos lucros, o custo exigido para a obtenção e a manutenção de um bom rebanho merece especial atenção dos produtores.

Os gerenciamentos do controle nutricional da novilha na sua fase de crescimento e do momento adequado para inseminação influenciam significativamente a rentabilidade da futura novilha leiteira [5]. Segundo Wattiaux [6], todo tipo de controle nessa atividade agropecuária é considerado essencial, visto que qualquer projeto de melhoria de uma propriedade leiteira necessita constantemente repor animais que são descartados em função da idade ou da baixa produtividade leiteira. Nessa perspectiva, o estabelecimento de um sistema de cria e recria eficiente para as fêmeas em rebanhos leiteiros é um desafio para a maioria dos produtores. As bezerras devem receber alimentação e manejo adequados para que possam atingir o peso ideal à primeira cobertura e iniciarem a sua vida produtiva o mais cedo possível.

O ideal é que o próprio produtor crie suas bezerras, pois, dessa forma, ele acompanhará a formação de seu rebanho, impedindo que se torne rotineira a criação e o desenvolvimento de animais de baixa qualidade, pois as bezerras que possui serão, em um futuro breve, as vacas leiteiras da propriedade. Dessa forma, é preciso cuidado para que as bezerras não sejam cobertas precocemente, sem que seu organismo esteja preparado para a reprodução, pois este fato pode interferir na 
quantidade do leite e no seu período de lactação. Quer dizer, o crescimento demasiadamente rápido, ou lento, traz prejuízos ao animal e ao criador. Assim, é imprescindível entender e acompanhar o crescimento da bezerra, do nascimento até o período de cobertura, que ocorre por volta de 18 meses.

Quanto aos aspectos reprodutivos, recomenda-se que a primeira inseminação ou cobertura deve ocorrer com o peso mínimo de $340 \mathrm{~kg}$ para bezerras da ração holandesa e de $230 \mathrm{~kg}$ para o gado Jersey. Se as novilhas forem acasaladas com baixo peso, terão que ganhar muito peso em pouco tempo, o que não é recomendado, ou irão parir com baixo peso, o que irá afetar a eficiência reprodutiva, a produção de leite e o crescimento do animal. Desse modo, para os produtores, é importante monitorar o animal para que este represente lucratividade ao longo de seu crescimento [3].

O controle de crescimento é considerado uma ferramenta na avaliação de bezerras, sendo um elemento determinante na produtividade e na rentabilidade do rebanho, exigindo maiores cuidados, pois envolve um alto custo de produção, visto que nesse período o animal não fornece nenhum retorno ao produtor. Esse controle permite a comparação do peso e da altura das bezerras, tendo por base o padrão de cada uma das raças. Com isso, o produtor consegue monitorar a taxa de crescimento das novilhas, balancear dietas de acordo com o estado nutricional do animal, dosar medicamentos e melhorar o potencial genético do rebanho. Para a elaboração desse controle, é avaliado o peso e a altura no decorrer do período de cria e recria [4].

Segundo Gomes [4], há uma variedade de métodos utilizados para a avaliação do peso vivo em novilhas, dentre eles, a balança calibrada ou a fita torácica de pesagem. O uso da balança calibrada não se mostra viável, em razão da dificuldade da contenção do animal, do tempo envolvido na atividade e de seu custo de implantação. A fita torácica, por sua vez, não é encontrada com facilidade nos estabelecimentos agropecuários. Nesse sentido, importante destacar que a medida do perímetro torácico pode predizer o peso do animal, refletindo no crescimento de órgãos, músculos e tecido adiposo. Essa medida vem sendo reconhecida por autoridades em gado leiteiro como um meio prático de estimar o peso vivo dos animais e, consequentemente, de ajudar a balancear suas dietas e acompanhar o seu crescimento.

Visando colaborar com esse processo de acompanhamento do peso do animal por meio de instrumentos mais fáceis de serem utilizados pelo produtor, apresentamos, a seguir, uma proposta de modelagem matemática que possibilita a estimativa do peso do animal a partir da medida torácica obtida com fita métrica comum.

\section{Metodologia}

A origem dos modelos e a história da modelagem encontram-se mergulhadas nos registros da ciência. Nesse sentido, destaca-se que, atualmente, encontram-se registros e conceitos variados para a modelagem, que pode ser entendida como a ação que objetiva a construção de modelos ou de processos utilizados na elaboração de um modelo, o qual pode ser concebido, em matemática, como um conjunto de símbolos que interagem entre si, representando alguma coisa. Essa representação pode se dar por meio de desenho ou imagem, projeto, esquema, gráfico, lei matemática, dentre outras formas [1], [2].

Para Bassanezi [1], o processo de modelagem consiste na arte de transformar problemas da realidade em problemas matemáticos e de resolvê-los interpretando suas soluções na linguagem do mundo real. Também focando a relação matemática e realidade, Biembengut [2] considera que a modelagem é como um meio para integrar esses dois conjuntos aparentemente disjuntos: matemática e realidade. Isso sugere traduzir a linguagem do mundo real para a linguagem do mundo matemático, ou seja, relacionar dois domínios ou mundos distintos.

Assim, a modelagem matemática possibilita que se descreva o peso vivo das bezerras em função de seu perímetro torácico, por meio de um modelo matemático, bem como permite que se compreenda as relações existentes entre eles. Além disso, constitui-se num instrumento que pode ser utilizado pelo produtor para o controle do crescimento de suas bezerras, sendo tal controle imprescindível no período de cria e recria, que corresponde do nascimento até, aproximadamente, os 18 meses do animal,. Após esse período, este instrumento não seria necessário, pois suas principais finalidades 
consistem em auxiliar o produtor a acompanhar e a controlar o peso das bezerras, otimizando as condições orgânicas destas para inseminação.

Para a escolha do modelo a ser utilizado para o ajuste, foram considerados os dados reais, constantes na Tabela 1, relativos ao crescimento padrão de perímetro torácico $(\mathrm{cm})$, peso $(\mathrm{kg})$ e altura $(\mathrm{cm})$ para bezerras da raça Holandesa.

\begin{tabular}{ccccccc}
\hline & \multicolumn{5}{c}{ JERSEY } & \multicolumn{3}{c}{ HOLANDESA } \\
\hline $\begin{array}{c}\text { Idade } \\
\text { (meses): }\end{array}$ & $\begin{array}{c}\text { Perímetro } \\
\text { torácico }\end{array}$ & Altura & Peso & $\begin{array}{c}\text { Perímetro } \\
\text { torácico }\end{array}$ & Altura & Peso \\
0 & 67 & & 24 & 70 & & 40 \\
1 & 73 & 70 & 30 & 86 & 80 & 55 \\
2 & 80 & 75 & 45 & 93 & 83 & 73 \\
3 & 86 & 80 & 60 & 102 & 87 & 94 \\
4 & 94 & 84 & 75 & 108 & 91 & 115 \\
5 & 100 & 90 & 92 & 115 & 95 & 136 \\
6 & 107 & 92 & 110 & 121 & 100 & 157 \\
7 & 114 & 95 & 128 & 127 & 104 & 180 \\
8 & 119 & 97 & 146 & 133 & 107 & 204 \\
9 & 124 & 99 & 162 & 139 & 110 & 228 \\
10 & 130 & 100 & 183 & 143 & 112 & 250 \\
11 & 134 & 103 & 193 & 148 & 115 & 273 \\
12 & 138 & 106 & 210 & 153 & 117 & 295 \\
13 & 141 & 107 & 225 & 157 & 119 & 317 \\
14 & 144 & 110 & 236 & 162 & 121 & 340 \\
15 & 148 & 112 & 255 & 165 & 123 & 360 \\
18 & 150 & 114 & 270 & 165 & 127 & 403 \\
\hline
\end{tabular}

Tabela 1: Dados relativos ao crescimento padrão de perímetro torácico $(\mathrm{cm})$, peso $(\mathrm{kg})$ e altura $(\mathrm{cm})$ para bezerras. FONTE: Projeto SEBRAE-ENARCAN

Com a utilização do Excel, utilizando os dados da Tabela 1, foi possível obter a visualização gráfica da curva aproximada, que descreve o comportamento real da variação do peso em relação à variação do perímetro torácico. Percebemos que, no intervalo de 0 a 18 meses, a curva obtida se assemelhava à curva geral que descreve o crescimento exponencial.

Essa análise nos possibilitou intuir qual deveria ser o modelo matemático que deveríamos escolher, de modo a realizar o ajuste de dados reais para que o modelo obtido pudesse traduzir de maneira coerente a realidade considerada.

Ressalta-se que a aproximação do peso em função do perímetro torácico foi considerada apenas no período entre 0 e 18 meses, visto que, após essa idade, o peso do animal oscilará em função de suas gestações e pós-gestações.

Assim, na modelagem proposta, nossa hipótese para descrever o comportamento do peso $(P)$ em função do perímetro torácico $(x)$ consiste em representá-lo por meio do modelo de crescimento exponencial [1], descrito pela seguinte equação diferencial ordinária: $\frac{d P}{d x}=k P$, que significa que partimos do pressuposto de que a variação do peso $(P)$ em relação à variação do perímetro $(x)$ seria proporcional ao peso $(P)$.

Para resolvê-la, considerando que $P=P(x)$ deva satisfazer uma condição em particular, devemos resolver o problema: 


$$
\left\{\begin{array}{l}
\frac{d P}{d x}=k P \\
P\left(x_{0}\right)=P_{0} \quad \text { (condição inicial) }
\end{array}\right.
$$

onde: $P_{0}$ é o peso no instante inicial e $x_{0}$ seria o perímetro no instante inicial.

A resolução da equação diferencial $\frac{d P}{d x}=k P$ fornece a solução de $P=P(x)$, que é obtida, após a separação de variáveis, por meio da integração da expressão: $\frac{1}{P} d P=k d x$, supondo $P \neq 0$. Dessa forma, resolvendo a equação diferencial e chamando $A=\frac{P\left(x_{0}\right)}{e^{k x_{0}}}$, temos: $P(x)=A e^{k x}$, que representa o modelo matemático para o crescimento exponencial escolhido, para descrever o comportamento do peso $(P)$ em função do perímetro torácico $(x)$.

Para a obtenção da estimativa dos parâmetros $A$ e $k$, utilizando o ajuste dos dados reais por meio do modelo de crescimento exponencial, consideramos que:

$$
\frac{d P}{d x}=k P \quad \quad \quad \text { ou seja: } \quad \frac{\Delta P}{\Delta x}=k P
$$

onde: $P$ : peso do animal no mês $i ; \Delta P=P_{i}-P_{i-1}$ : variação dos pesos, do mês $i-1$ até o mês $i$ e $\Delta x=x_{i}-x_{i-1}$ : variação dos perímetros, do mês $i-1$ até o mês $i$.

Assim, foi possível calcular o valor de $k_{i}=\frac{1}{P} \frac{\Delta P}{\Delta x}$, para cada intervalo considerado.

Além disso, também foi considerado o valor médio de $\bar{k}$, dado por: $\bar{k}=\frac{\sum_{i=1}^{n} k_{i}}{n}$, o peso $P\left(x_{i}\right)$ e o perímetro $x_{i}$ para o mês $i$, para se calcular o valor aproximado de $A$ ( chamado de $\bar{A}$ ), calculado pela seguinte expressão: $\bar{A}=\frac{\sum_{i=1}^{n} A_{i}}{n}, \quad$ onde: $A_{i}=\frac{P\left(x_{i}\right)}{e^{\bar{k} x_{i}}}$.

Esses ajustes possibilitam calcular os parâmetros ajustados do modelo para o crescimento exponencial, o que resultou em: $P(x)=\bar{A} e^{\bar{k} x}$.

\section{Resultados obtidos}

Para a validação do modelo obtido pelo ajuste proposto, foram considerados dados reais, para o gado holandês, apresentados na Tabela 1.

Após a escolha do modelo de crescimento exponencial e dos ajustes para o cálculo de seus parâmetros, considerando os dados da Tabela 1, foram calculados os parâmetros aproximados para o modelo de crescimento exponencial proposto, os quais foram obtidos por meio de uma planilha do Excel. Tais dados são apresentados na Tabela 2.

Substituindo os valores calculados em: $P(x)=\bar{A} e^{\bar{k} x}$, obtém-se o modelo exponencial aproximado, que descreve a relação entre a variação do peso em função do perímetro torácico dado por: $P(x)=13,1188 e^{0,02 x}$, cujo gráfico pode ser visualizado na Figura 1. 
A comparação entre os valores reais e os valores aproximados para a validação do modelo proposto pode ser realizada tanto pela análise gráfica, pela observação da Figura 1, quanto pela comparação dos valores reais e aproximados, apresentados na Tabela 3.

\begin{tabular}{|c|c|c|c|c|c|c|}
\hline \multicolumn{7}{|c|}{ Gado holandês } \\
\hline $\begin{array}{l}\text { Idade } \\
\text { (meses) }\end{array}$ & $\begin{array}{l}x: \text { Perímetro } \\
\text { torácico }(\mathrm{cm})\end{array}$ & $\begin{array}{c}P: \text { Peso } \\
(\mathrm{kg})\end{array}$ & $\Delta x$ & $\Delta P$ & $k_{i}=\frac{1}{P} \frac{\Delta P}{\Delta x}$ & $A_{i}=\frac{P\left(x_{i}\right)}{e^{\bar{K} x_{i}}}$ \\
\hline 0 & 70 & 40 & & & & \\
\hline 1 & 86 & 55 & 16 & 15 & 0,0170 & 9,8337 \\
\hline 2 & 93 & 73 & 7 & 18 & 0,0352 & 11,3455 \\
\hline 3 & 102 & 94 & 9 & 21 & 0,0248 & 12,2007 \\
\hline 4 & 108 & 115 & 6 & 21 & 0,0304 & 13,2372 \\
\hline 5 & 115 & 136 & 7 & 21 & 0,0221 & 13,6076 \\
\hline 6 & 121 & 157 & 6 & 21 & 0,0223 & 13,9309 \\
\hline 7 & 127 & 180 & 6 & 23 & 0,0213 & 14,1642 \\
\hline 8 & 133 & 204 & 6 & 24 & 0,0196 & 14,2360 \\
\hline 9 & 139 & 228 & 6 & 24 & 0,0175 & 14,1102 \\
\hline 10 & 143 & 250 & 4 & 22 & 0,0220 & 14,2811 \\
\hline 11 & 148 & 273 & 5 & 23 & 0,0168 & 14,1097 \\
\hline 12 & 153 & 295 & 5 & 22 & 0,0149 & 13,7946 \\
\hline 13 & 157 & 317 & 4 & 22 & 0,0174 & 13,6827 \\
\hline 14 & 162 & 340 & 5 & 23 & 0,0135 & 13,2778 \\
\hline 15 & 165 & 360 & 3 & 20 & 0,0185 & 13,2394 \\
\hline 18 & 175 & 403 & 10 & 43 & 0,0107 & 12,1321 \\
\hline 24 & 188 & 510 & 13 & 107 & 0,0161 & 11,8354 \\
\hline \multicolumn{5}{|c|}{ Valores médios } & $\bar{k}=0,0200$ & $\bar{A}=13,1188$ \\
\hline
\end{tabular}

Tabela 2: Tabela de ajuste pelo modelo de crescimento exponencial proposto

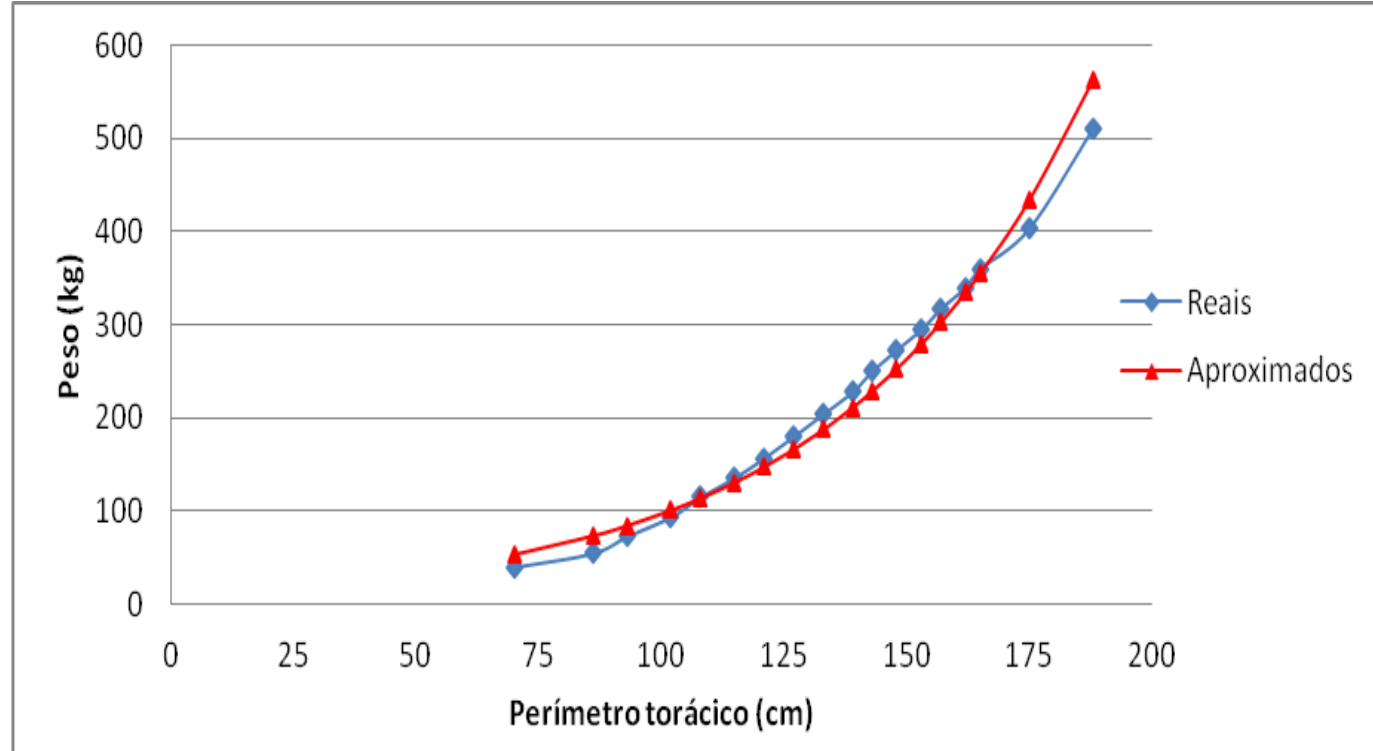

Figura 2: Representação gráfica do peso $(\mathrm{kg})$ real e aproximado em função do perímetro torácico das bezerras. 
Desenvolvemos, portanto, um instrumento, elaborado no ambiente do Excel, que possibilita estimar e comparar pesos aproximados para novos perímetros torácicos informados, sendo os resultados gerados pelo modelo exponencial proposto, com os valores dos pesos reais, disponíveis na literatura, conforme pode ser visualizado na Figura 3.

Acreditamos que este instrumento pode ser disponibilizado aos criadores, de modo a possibilitar a estimativa de peso das bezerras apenas com auxílio de um computador e de uma fita métrica comum. Nessa perspectiva, há que se destacar que sua principal utilidade consiste em facilitar, para produtores que não disponham nem de balança, nem de fita torácica, o acompanhamento do crescimento das bezerras.

\begin{tabular}{cccc}
\hline Idade $($ meses $)$ & $\begin{array}{c}\text { x: Perímetro } \\
\text { torácico }(\mathrm{cm})\end{array}$ & P: Peso $(\mathrm{kg})$ & $\begin{array}{c}\text { Peso aprox. } \\
(\mathrm{kg})\end{array}$ \\
\hline 0 & 70 & 40 & 53,20 \\
1 & 86 & 55 & 73,26 \\
2 & 93 & 73 & 84,27 \\
3 & 102 & 94 & 100,89 \\
4 & 108 & 115 & 113,75 \\
5 & 115 & 136 & 130,85 \\
6 & 121 & 157 & 147,53 \\
7 & 127 & 180 & 166,34 \\
8 & 133 & 204 & 187,55 \\
9 & 139 & 228 & 211,46 \\
10 & 143 & 250 & 229,07 \\
11 & 148 & 273 & 253,17 \\
12 & 153 & 295 & 279,79 \\
13 & 157 & 317 & 303,10 \\
14 & 162 & 340 & 334,97 \\
15 & 165 & 360 & 355,69 \\
18 & 175 & 403 & 434,43 \\
\hline
\end{tabular}

Tabela 3: Validação do modelo - dados reais e aproximados (obtidos por meio do modelo considerado).

\section{Considerações finais}

A modelagem matemática, por meio de modelos analógicos, possibilita a estimativa de valores aproximados para dados em situações reais, os quais, muitas vezes, são fundamentais para o planejamento de ações que podem auxiliar gestores no processo administrativo de tomada de decisão.

Neste artigo, pelos resultados obtidos, percebemos que a hipótese de ajuste dos dados por meio do modelo exponencial, para os meses de crescimento da bezerra até o período de cobrimento, foi adequada, tendo em vista que os pesos aproximados calculados ficaram bastante próximos dos pesos reais, disponíveis na literatura.

Acreditamos que a proposta de modelagem apresentada, para estimativa de peso de bezerras em função do perímetro torácico obtido por fita métrica comum, pode ser uma inovação nessa área agropecuária, pois possibilita a utilização de instrumentos de fácil manejo para o acompanhamento do crescimento das bezerras, pelos seus produtores. Auxilia ainda os interessados na área zootécnica na manutenção de um bom rebanho, possibilitando o balanceamento de dietas ao longo do período de crescimento e antes do período de cobertura, de modo que o rebanho possa receber alimentação e manejo adequados para que possa atingir o peso ideal para a primeira cobertura. 


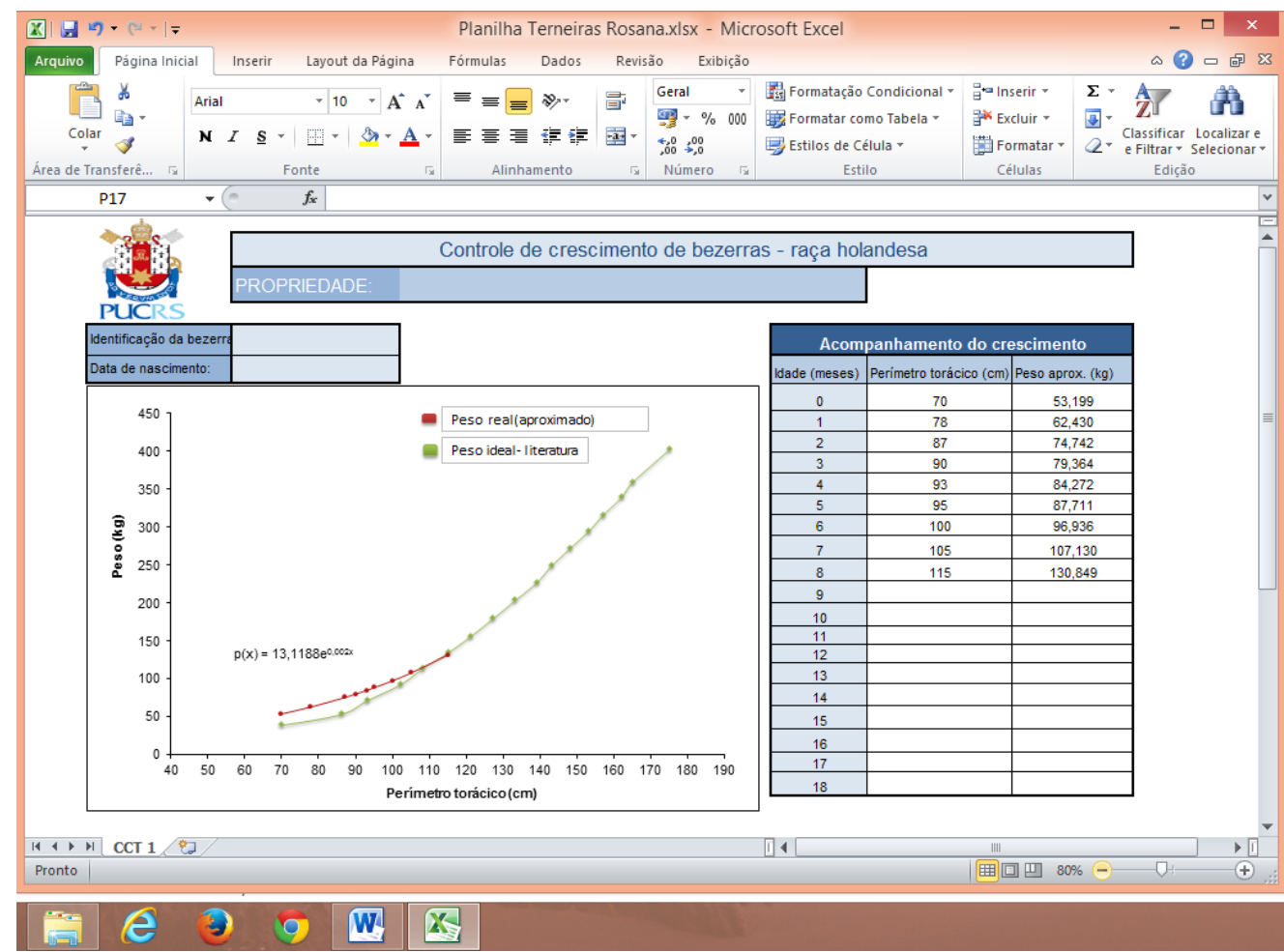

Figura 3: Instrumento para controle de crescimento das bezerras, que considera o ajuste proposto para o modelo exponencial considerado.

\section{Referências}

[1] R. C. Bassanezi, "Ensino-aprendizagem com modelagem matemática". 3. ed. São Paulo: Contexto, 2006.

[2] M. S. Biembengut, "Modelagem matemática \& Implicações no Ensino e na Aprendizagem de Matemática". 2. ed. Blumenau: Edifurb, 2004.

[3] O. F Campos; R. S Lizieire. "Criação de bezerras e novilhas em sistemas de produção de leite". Disponível em:

<http://www.cnpgl.embrapa.br/totem/conteudo/Alimentacao_e_manejo_animal/Outras_publicacoes/C riacao_de_bezerras_e_novilhas_em_sistemas_de_producao_de_leite.pdf.>. Acesso em: 10 set. 2013.

[4] R. Gomes. “A bezerra de hoje é a vaca do futuro". 2011. ABS América Latina. Disponível em: <www.absamericalatina.com> Acesso em: 12 set. 2013.

[5] M.C.M. Mourits, R.B.M. Huime, A.A. Dijkhuizen, A.R. Kristensen, D.T. Galligan. Economic optimization of dairy heifer management decisions. Agricultural Systems, vol. 61, pp. 17-31, (1999)

[6] A. M. Wattiaux, "Criação de novilhas desmama ao primeiro parto, taxa do controle de crescimento". Instituto Babcock para Pesquisa e Desenvolvimento da Pecuária Leiteira Internacional University of Wisconsin-Madison, 2007. 